\title{
Retrospective analysis of the role of intra-operative parathyroid hormone monitoring during parathyroidectomy for primary hyperparathyroidism; a single center experience over 2 decades
}

\author{
Yehonatan Adler ${ }^{1}$, Sharon Tzelnick ${ }^{1}$, Yoni Shopen ${ }^{1}$, Ella Reifen ${ }^{1}$, Gideon Bachar ${ }^{1}$, Thomas \\ Shpitzer ${ }^{1}$, and Aviram Mizrachi ${ }^{1}$ \\ ${ }^{1}$ Rabin Medical Center
}

December 7, 2021

\begin{abstract}
Background: The role of intra-operative parathyroid hormone (IOPTH) monitoring during parathyroidectomy for primary hyperparathyroidism has long been debated. Objectives: Our main goal was to investigate the cure rates of parathyroidectomy for primary hyperparathyroidism with and without IOPTH monitoring. Our secondary goal was to investigate if operating room time can be saved when not using IOPTH monitoring. Design: A retrospective analysis of patients who underwent parathyroidectomy for PHPT for a single adenoma between 2004-2019 was performed. Cure rates and operating room time were compared. Results: 423 patients were included. IOPTH was used in 248 patients (59\%). Four patients were not cured, two from each group, with no significant difference between the groups $(98.8 \%$ vs. $99.1 \%$, p=0.725). Surgery time was significantly longer in the IOPTH group, $\mathrm{p}<0.001$. Conclusions: There is no advantage for using IOPTH during parathyroidectomy in suitable clinical setting. A focused procedure may be safely performed without IOPTH while achieving non-inferior success rates and reducing operative time.
\end{abstract}

\section{ABSTRACT}

Background: The role of intra-operative parathyroid hormone (IOPTH) monitoring during parathyroidectomy for primary hyperparathyroidism has long been debated.

Objectives : Our main goal was to investigate the cure rates of parathyroidectomy for primary hyperparathyroidism with and without IOPTH monitoring. Our secondary goal was to investigate if operating room time can be saved when not using IOPTH monitoring.

Design : A retrospective analysis of patients who underwent parathyroidectomy for PHPT for a single adenoma between 2004-2019 was performed. Cure rates and operating room time were compared.

Results : 423 patients were included. IOPTH was used in 248 patients (59\%). Four patients were not cured, two from each group, with no significant difference between the groups ( $98.8 \%$ vs. $99.1 \%, \mathrm{p}=0.725)$. Surgery time was significantly longer in the IOPTH group, $\mathrm{p}<0.001$.

Conclusions: There is no advantage for using IOPTH during parathyroidectomy in suitable clinical setting. A focused procedure may be safely performed without IOPTH while achieving non-inferior success rates and reducing operative time.

\section{Keywords}

Parathyroidectomy, Primary Hyperparathyroidism, Intra-Operative Parathyroid Hormone Monitoring, Parathyroid Adenoma, Operative Time 


\section{KEY POINTS}

- IOPTH monitoring is a long-debated topic. Some advocate its use only in specific clinical scenarios, while others advocate its use in every focused parathyroidectomy.

- There was no significant difference in surgical success rates between the "IOPTH used" and the "IOPTH not used" groups.

- Operating room time was significantly shorter in the "IOPTH not used" group.

- When two imaging modalities are concordant for the location of a single adenoma, it appears that a focused parathyroidectomy without IOPTH monitoring can be used safely.

- IOPTH monitoring should be used only in select cases.

\section{INTRODUCTION}

Primary Hyperparathyroidism (PHPT), a benign hormonal disorder characterized by a high serum calcium concentration and an inappropriately increased parathyroid hormone, is a relatively common medical condition, with prevalence of 233 to 100,000 in women, and 85 to 100,000 in men. ${ }^{(1)}$ Approximately $88 \%$ of cases are due to single adenoma. ${ }^{(2)}$

The treatment of choice for PHPT is parathyroidectomy with a cure rate of up to $99 \%{ }^{(3-7)}$

Over the last few decades, the minimally invasive parathyroidectomy has gradually replaced the standard procedure - bilateral neck exploration ${ }^{(8)}$ - due to preoperative localization techniques and intraoperative parathyroid hormone monitoring (IOPTH).

Although preoperative localization techniques vary, Ultrasonography (US) and technetium Sestamibi scintigraphy (Sestamibi) reach a high sensitivity rate of up to $90 \% .^{(6,9-11)}$ Other imaging modalities, such as Computed Tomography (CT) and MRI, are acceptable as well.

The definitive treatment for PHPT due to single adenoma, is resection of the affected gland. Cure is defined as a decline in calcium levels to normal $(8.4-10.5 \mathrm{mg} / \mathrm{dL})$, six months post-surgery.

Pre-operative imaging for adenoma localization varies between centers worldwide.

The routine use of IOPTH is a long-debated topic in the literature. In a recent survey in the United States, $74 \%$ of surgeons responded they always use IOPTH, regardless of the clinical setting. ${ }^{(12)}$

The American Association of Endocrine Surgeons recommends performing IOPTH in all parathyroidectomies. ${ }^{(13)}$ Several studies have shown that the use of IOPTH results in cure rates of 97-99\%. ${ }^{(3-5)}$ Furthermore, studies have also shown that when IOPTH is not performed, there is a decline in operative success. ${ }^{(5,13,14)}$

Notwithstanding the above, the European Society of Endocrine Surgeons (ESES) recommends that when pre-operative localization with Sestamibi and US is concordant for single gland disease, the use of IOPTH is of little added value (evidence at levels Ib-III, recommendation grades A/B). ${ }^{(15)}$ Other studies have shown that when two imaging modalities are concordant pre-operatively, there is no difference in cure rates when IOPTH is not used. ${ }^{(6,16,17)}$ In addition, some studies have shown that unrelated to imaging modalities, IOPTH usage does not change the surgical success rates. ${ }^{(18-20)}$ Furthermore, in a study that examined 15,000 parathyroidectomies, the authors argue that even $90 \%$ decline in IOPTH monitoring during surgery, cannot assure cure. They suggest that all parathyroidectomies should be done with bilateral exploration, without IOPTH monitoring. ${ }^{(21)}$

Regarding operating room time and operating room economics, a comprehensive cost analysis published in 2010 showed that many parameters should be taken into consideration when assessing the cost-benefit of using IOPTH, the most important of which is the rate of multi-glandular disease ${ }^{(22)}$

A decade ago, a study published by our group demonstrated no added value for IOPTH when US and MIBI are concordant. ${ }^{(6)}$ Since then, we have changed our practice and utilized IOPTH only in selected 
cases (pre-operative imaging is not concordant; suspected multiglandular disease; secondary or tertiary hyperparathyroidism; patients with a diagnosis of MEN1 or MEN2A; revision surgery).

The main goal of the present study was to revisit our protocol following 10 years of experience, by investigating the cure rates of primary hyperparathyroidism since the protocol had changed, compared to the period when IOPTH was used routinely. The secondary goal was to investigate if operating room time can be saved when IOPTH is not used.

\section{MATERIALS AND METHODS}

\section{Study Design}

A retrospective analysis of all patients who underwent parathyroidectomy for primary hyperparathyroidism at a university-affiliated tertiary care center between 2004-2019 was performed. Patients were followed for a minimum of 6 months. Subsequently, a comparison of the cure rates (normalization of serum calcium levels at 6 months post-surgery) was made between patients who underwent parathyroidectomy with IOPTH and those who underwent parathyroidectomy without IOPTH. Only patients who underwent focused parathyroidectomy were included in the study. Operating room time was also compared between the two groups.

The study was approved by the Institutional Review Board.

\section{Rabin Medical Center parathyroidectomy protocol}

Since 2010, our protocol at Rabin medical center for parathyroidectomies is as follows: localization of a parathyroid adenoma is performed for all patients using two imaging modalities: Sestamibi and US. If both imaging modalities are concordant, confirmation of adenoma resection during surgery is performed with frozen section, without the use of IOPTH monitoring. We do use IOPTH monitoring during parathyroidectomy in any other clinical scenario, such as: pre-operative imaging is not concordant; double adenoma; parathyroid hyperplasia; secondary or tertiary hyperparathyroidism; patients with a diagnosis of MEN1 or MEN2A; revision surgery.

Pre-operative adenoma localization

Pre-operative adenoma localization modalities included both US, and Sestamibi. All localization studies were performed and analyzed by specialized, experienced radiologists.

\section{Operating room time}

Operating room time was measured in minutes, from first incision to final suture.

IOPTH assay and Protocol

We used the assay "Elecsys PTH (1-84)" by Roche (Switzerland) and follow the Miami protocol for IOPTH monitoring. ${ }^{(23)}$

Statistical Analysis

Data analysis was performed with SPSS.

Descriptive statistics : Categorical variables were expressed as frequency and percentages. Continuous variables were expressed as mean and standard deviation (SD).

Univariate analysis : differences between the two groups in continuous variables with normally distributed variables ware analyzed with student's T-test. Non normally distributed variables were analyzed with Mann Whitney U test. Differences between the two groups in categorical variables was analyzed with Chi-Squared test.

\section{RESULTS}

Overall, 452 patients underwent surgery in the above-mentioned period. A total of 29 patients were excluded from the study: 17 patients were excluded due to suspected multiglandular disease preoperatively; 11 patients 
were excluded due to insufficient data regarding their cure rates or loss to follow-up; 1 patient was excluded due to adenoma resection using a different surgical modality (Da-Vinci).

A total of 423 patients were included in the analysis, 175 in the No IOPTH group and 248 in the IOPTH group. Table 1 presents comparison of demographic and clinical data between the two groups. Although two statistically significant differences were found between the groups (PTH Pre-Op and Calcium Pre-Op), both are clinically insignificant.

Pre-operative concordance between US and Sestamibi in all patients was $76 \%$. The positive predictive value of both modalities for the location of the adenoma as demonstrated during surgery and after the final pathologic report was $90 \%$, and the sensitivity was $74 \%$.

A total of $173(98.8 \%)$ patients from the No IOPTH group demonstrated normalization of calcium levels 6 months after surgery. A similar cure rates were noted in the IOPTH group (246 patients with a cure rate of $99.1 \%)$. Only 2 patients from each group were not cured $(\mathrm{p}=0.725)$.

The mean operative time was 66.52 minutes $( \pm 28.145)$ in the No IOPTH group, $86.97( \pm 32.353)$ minutes in the IOPTH group, $88.28( \pm 29.594)$ minutes in the IOPTH-NC group and $86.35( \pm 33.633)$ in the IOPTH-C group.

Operative time was significantly longer in the IOPTH group compared to the No IOPTH group $(\mathrm{p}<0.001)$. Furthermore, when comparing operative time between the No IOPTH group and the two subgroups (IOPTH$\mathrm{NC}$ and IOPTH-C), we found that operative time was significantly longer in both subgroups regardless of imaging concordance $(\mathrm{p}<0.001)$ (Figure 1).

\section{DISCUSSION}

In our study, we aimed to investigate the differences in cure rate and operative time, using IOPTH. We have shown, in a large cohort, that there is no statistical difference in cure rate with or without IOPTH assay, and that using IOPTH significantly increases operative time.

This study was conducted as a follow-up to a previous study we performed in 2010. ${ }^{(6)}$ Following the 2010 study, our department has changed its practice and stopped using IOPTH routinely in every parathyroidectomy, but only in selected clinical scenarios, as outlined above.

IOPTH assay is performed routinely by $74 \%$ of parathyroid surgeons in the US. ${ }^{(12)}$ However, its routine use in all parathyroidectomies is long debated.

There is a wide consensus amongst most parathyroid surgeons and the literature that several scenarios necessitate its use, such as unconcordant pre-operative localization studies or multiglandular disease, and both the ESES (European Society of Endocrine Surgeons) and the AAES (American Association of Endocrine Surgeons) agree on its use in such circumstances.

Whilst the clinical scenarios are agreed upon, the routine use of IOPTH in every parathyroidectomy, regardless of the scenario, is widely debated. Two main approaches in the literature that represent this debate, are the ESES and the AAES: the ESES recommends that "when preoperative localization with MIBI and US is concordant for single-gland disease, the use of IOPTH is of little added value"; on the other hand, the AAES recommends that when image-guided focused Parathyroidectomy is planned, IOPTH should be used to avoid higher operative failure rates. ${ }^{(13,15)}$

Another noteworthy approach is that of J. Norman et al. who advocated to abandon unilateral parathyroidectomies and perform a bilateral exploration in every parathyroidectomy.(21) However, it should be emphasized, that their group does not use US at all, but rather Sestamibi as the single pre-operative localization modality.

At Rabin Medical Center, we follow the ESES recommendation, with a 99\% success rate without the routine use of IOPTH. Our approach for primary hyperparathyroidism due to a single adenoma with two concordant imaging modalities for pre-operative localization, is minimally invasive surgery, performed by experienced surgeons and a frozen section confirmation. 
According to the literature, the sensitivity and PPV of pre-operative localization using both Sestamibi and US, ranges from $78.7-91.9 \%$ and $89.8-100 \%$ respectively. ${ }^{(24)}$ Our results are on the lower side of these ranges (sensitivity: $74.17 \%$, PPV: $90.06 \%$ ), and are lower than the rates we have shown in a previous study conducted at our center. We speculate that several factors contributed to these rates: (1) Regarding the low sensitivity, whenever the two modalities were unconcordant, even if one of them showed no adenoma while the other predicted the location of the adenoma correctly, we labeled it as "False Negative"; (2) Not all US and Sestamibi tests were performed by the same operator, and many of them were not performed by experts specialized solely in head \& neck imaging. In a previous study performed at our center, we included mainly patients who underwent US and Sestamibi by a specialized head \& neck operator. Sensitivity and PPV were $93 \%$ and $99 \%$ respectively $^{(6)}$

Regarding operative time analysis, we decided to divide our control group into two subgroups as outlined in the "Methods" section. We wanted to examine the hypothesis that because many patients who underwent parathyroidectomy with IOPTH monitoring had unconcordant pre-operative localization, their operative time would be longer a priori, thus leading to a bias when comparing operative time between the study and control group. We have shown, that regardless of pre-operative localization studies concordance, operative time with IOPTH monitoring is significantly longer compared to operative time without IOPTH monitoring.

Economically, the IOPTH assay affects operative financial costs, primarily in two manners: the first is the cost of operating room time, which was significantly shorter in our No IOPTH group compared individually to both IOPTH subgroups; the second is the direct cost of the assay itself.

In a study conducted L. Morris et $\mathrm{al}^{(23)}$, the authors performed a cost-benefit analysis of IOPTH use. For them, when considering the cost-benefit of routine IOPTH use, the most important factor to weigh, is the rate of multi-glandular disease, which varies substantially amongst different institutions and countries. They argue that when pre-operatively unrecognized multi-glandular disease exceeds $6 \%$, it is reasonable to perform IOPTH in all Parathyroidectomies. In our cohort only 3.7\% (17/452) of patients had a multi-glandular disease discovered intra-operatively. Additionally, the authors argue that IOPTH use becomes cost-saving when the probability of cure without IOPTH monitoring decreases below $94 \%$. In our study we reached a $98.8 \%$ cure rate in the No IOPTH group.

Likewise, as shown in a study by Badii B et al. from 2017, when IOPTH is not used routinely in every parathyroidectomy, but only in selected patients, a substantial amount of money can be saved - up to leuro678 per patient. ${ }^{(25)}$

Moreover, as health-economics become a substantial consideration in modern medical centers, a protocol to reduce costs, without diminishing the success rates of a commonly performed procedure, is paramount.

Based on this study, we would like to propose that using our protocol for parathyroidectomies is non-inferior to parathyroidectomies performed with IOPTH assay, while also saving operating room time.

The present study is limited by its retrospective nature and the fact that the data had been collected over almost two decades. Nevertheless, the study timeline reflects the natural evolution of IOPTH and the transition to a minimally invasive approach. Furthermore, the importance of continuous report from a single high-volume center cannot be overstated, since practice change needs to be reviewed respectively to assure quality.

\section{CONCLUSIONS}

The present study sheds light on the long-debated topic of routine IOPTH monitoring use. We continue to advocate using IOPTH only in selected patients with discordant preoperative localization of suspected multiglandular disease.

\section{CONFLICT OF INTERESTS}

All authors state that they have no conflict of interest to declare. 


\section{DATA AVAILABILITY STATEMENT}

The data that support the findings of this study are available on request from the corresponding author. The data are not publicly available due to privacy or ethical restrictions.

\section{REFERENCES}

1. Yeh MW, Ituarte PHG, Zhou HC, Nishimoto S, Liu I-LA, Harari A, et al. Incidence and prevalence of primary hyperparathyroidism in a racially mixed population. J Clin Endocrinol Metab. 2013 Mar;98(3):11229.

2. Ruda JM, Hollenbeak CS, Stack BC. A systematic review of the diagnosis and treatment of primary hyperparathyroidism from 1995 to 2003. Otolaryngol-Head Neck Surg Off J Am Acad Otolaryngol-Head Neck Surg. 2005 Mar;132(3):359-72.

3. Day KM, Elsayed M, Monchik JM. No Need to Abandon Focused Unilateral Exploration for Primary Hyperparathyroidism with Intraoperative Monitoring of Intact Parathyroid Hormone. J Am Coll Surg. 2015 Aug;221(2):518-23.

4. Udelsman R, Lin Z, Donovan P. The superiority of minimally invasive parathyroidectomy based on 1650 consecutive patients with primary hyperparathyroidism. Ann Surg. 2011 Mar;253(3):585-91.

5. Carneiro-Pla DM, Solorzano CC, Irvin GL. Consequences of targeted parathyroidectomy guided by localization studies without intraoperative parathyroid hormone monitoring. J Am Coll Surg. 2006 May;202(5):715-22.

6. Bachar G, Mizrachi A, Hadar T, Feinmesser R, Shpitzer T. Role of parathyroid hormone monitoring during parathyroidectomy. Head Neck. 2011 Dec;33(12):1754-7.

7. Gawande AA, Monchik JM, Abbruzzese TA, Iannuccilli JD, Ibrahim SI, Moore FD. Reassessment of parathyroid hormone monitoring during parathyroidectomy for primary hyperparathyroidism after 2 preoperative localization studies. Arch Surg Chic Ill 1960. 2006 Apr;141(4):381-4; discussion 384.

8. Grant CS, Thompson G, Farley D, van Heerden J. Primary hyperparathyroidism surgical management since the introduction of minimally invasive parathyroidectomy: Mayo Clinic experience. Arch Surg Chic Ill 1960. 2005 May;140(5):472-8; discussion 478-479.

9. Levy JM, Kandil E, Yau LC, Cuda JD, Sheth SN, Tufano RP. Can ultrasound be used as the primary screening modality for the localization of parathyroid disease prior to surgery for primary hyperparathyroidism? A review of 440 cases. ORL J Oto-Rhino-Laryngol Its Relat Spec. 2011;73(2):116-20.

10. Bhansali A, Masoodi SR, Bhadada S, Mittal BR, Behra A, Singh P. Ultrasonography in detection of single and multiple abnormal parathyroid glands in primary hyperparathyroidism: comparison with radionuclide scintigraphy and surgery. Clin Endocrinol (Oxf). 2006 Sep;65(3):340-5.

11. Stern S, Tzelnick S, Mizrachi A, Cohen M, Shpitzer T, Bachar G. Accuracy of Neck Ultrasonography in Predicting the Size and Location of Parathyroid Adenomas. Otolaryngol-Head Neck Surg Off J Am Acad Otolaryngol-Head Neck Surg. 2018 Dec;159(6):968-72.

12. Coan KE, Yen TWF, Carr AA, Evans DB, Wang TS. Confirmation of Parathyroid Tissue: Are Surgeons Aware of New and Novel Techniques? J Surg Res. 2020 Feb;246:139-44.

13. Bilezikian JP, Brandi ML, Eastell R, Silverberg SJ, Udelsman R, Marcocci C, et al. Guidelines for the management of asymptomatic primary hyperparathyroidism: summary statement from the Fourth International Workshop. J Clin Endocrinol Metab. 2014 Oct;99(10):3561-9.

14. Riss P, Scheuba C, Asari R, Bieglmayer C, Niederle B. Is minimally invasive parathyroidectomy without QPTH monitoring justified? Langenbecks Arch Surg. 2009 Sep;394(5):875-80. 
15. Bergenfelz AOJ, Hellman P, Harrison B, Sitges-Serra A, Dralle H, European Society of Endocrine Surgeons. Positional statement of the European Society of Endocrine Surgeons (ESES) on modern techniques in pHPT surgery. Langenbecks Arch Surg. 2009 Sep;394(5):761-4.

16. Helbrow J, Owais AE, Sidwell AG, Frank LM, Lucarotti ME. The use of intraoperative parathyroid hormone monitoring in minimally invasive parathyroid surgery. Ann R Coll Surg Engl. 2016 Sep;98(7):5169.

17. Sartori PV, Saibene AM, Leopaldi E, Boniardi M, Beretta E, Colombo S, et al. Intraoperative parathyroid hormone testing in primary hyperparathyroidism surgery: time for giving up? Eur Arch Oto-Rhino-Laryngol Off J Eur Fed Oto-Rhino-Laryngol Soc EUFOS Affil Ger Soc Oto-Rhino-Laryngol - Head Neck Surg. 2019 Jan;276(1):267-72.

18. Suliburk JW, Sywak MS, Sidhu SB, Delbridge LW. 1000 minimally invasive parathyroidectomies without intra-operative parathyroid hormone measurement: lessons learned. ANZ J Surg. 2011 May;81(5):362-5.

19. Najafian A, Kahan S, Olson MT, Tufano RP, Zeiger MA. Intraoperative PTH May Not Be Necessary in the Management of Primary Hyperparathyroidism Even with Only One Positive or Only Indeterminate Preoperative Localization Studies. World J Surg. 2017 Jun;41(6):1500-5.

20. Nair CG, Babu MJC, Jacob P, Menon R, Mathew J. Is intraoperative parathyroid hormone monitoring necessary in symptomatic primary hyperparathyroidism with concordant imaging? Indian J Endocrinol Metab. 2016 Aug;20(4):512-6.

21. Norman J, Lopez J, Politz D. Abandoning unilateral parathyroidectomy: why we reversed our position after 15,000 parathyroid operations. J Am Coll Surg. 2012 Mar;214(3):260-9.

22. Carneiro DM, Solorzano CC, Nader MC, Ramirez M, Irvin GL. Comparison of intraoperative iPTH assay (QPTH) criteria in guiding parathyroidectomy: which criterion is the most accurate? Surgery. 2003 Dec;134(6):973-9; discussion 979-981.

23. Morris LF, Zanocco K, Ituarte PHG, Ro K, Duh Q-Y, Sturgeon C, et al. The value of intraoperative parathyroid hormone monitoring in localized primary hyperparathyroidism: a cost analysis. Ann Surg Oncol. 2010 Mar;17(3):679-85.

24. Wang TS, Cheung K, Farrokhyar F, Roman SA, Sosa JA. Would scan, but which scan? A cost-utility analysis to optimize preoperative imaging for primary hyperparathyroidism. Surgery. 2011 Dec;150(6):128694.

25. Badii B, Staderini F, Foppa C, Tofani L, Skalamera I, Fiorenza G, et al. Cost-benefit analysis of the intraoperative parathyroid hormone assay in primary hyperparathyroidism. Head Neck. 2017 Feb;39(2):2416 .

\section{Hosted file}

Table 1 IOPTH.docx available at https://authorea.com/users/450021/articles/548457retrospective-analysis-of-the-role-of-intra-operative-parathyroid-hormone-monitoringduring-parathyroidectomy-for-primary-hyperparathyroidism-a-single-center-experienceover-2-decades

\section{Hosted file}

Figure 1 IOPTH.docx available at https://authorea.com/users/450021/articles/548457retrospective-analysis-of-the-role-of-intra-operative-parathyroid-hormone-monitoringduring-parathyroidectomy-for-primary-hyperparathyroidism-a-single-center-experienceover-2-decades 